\title{
Nonlinear QED in an ultrastrong rotating electric field: Signatures of the momentum-dependent effective mass
}

\author{
E. Raicher* and K. Z. Hatsagortsyan \\ Max-Planck-Institut für Kernphysik, Saupfercheckweg 1, 69117 Heidelberg, Germany
}

(Received 23 July 2019; accepted 16 January 2020; published 2 March 2020)

\begin{abstract}
The electron effective mass in a rotating electric field (REF) and its signatures in the nonlinear Breit-Wheeler and nonlinear Compton processes are investigated, recognizing this field as a model for the antinodes of counterpropagating laser beams. In contrast to the case of a plane-wave laser field, the effective mass in REF depends on the electron momentum absolute value as well as on its direction with respect to the field plane. These features of the effective mass are observable in the threshold for pair production by a $\gamma$ photon, and in the harmonics structure appearing in the radiation spectrum of an electron. It can be tested varying the photon propagation direction in the first case, and the electron energy in the second case. Thus, we demonstrate the possibility of a nontrivial dispersion relation for an electron in strong laser fields, analogous to that in condensed-matter systems, which leaves its imprints in high-energy scattering.
\end{abstract}

DOI: 10.1103/PhysRevResearch.2.013240

\section{INTRODUCTION}

The modification of a particle mass due to an interaction with a strong field is a fundamental phenomenon, appearing in a variety of fields in physics. In particle physics, the spontaneous electroweak symmetry breaking is accompanied by a nonzero Higgs field that endows the elementary particles with their mass [1]. In QCD, a quark gains an effective mass (known as a constituent mass) due to the field associated with chiral symmetry breaking [2]. The emergence of an effective mass is abundant in condensed-matter systems. As opposed to the above examples, however, in this case the external potential is periodic, resulting in a nontrivial modification of the electron dispersion relation. For instance, an electron in a lattice may exhibit a rich band structure, depending on its momentum and direction with respect to the crystal planes [3].

An effective mass arises also in the interaction between a high-intensity laser and a particle [4,5], stemming from the pondermotive potential associated with the laser [6]. The effective mass, rather than a bare one, appears in the energy momentum conservation law [7], thus leaving signatures in the corresponding nonlinear QED processes. From a theoretical point of view, the investigation of the effective mass associated with strong-field QED requires a solution for the dynamics of the particle in the presence of the field. Most of the analyses to be found in the literature were done for a particle in a plane-wave field (PWF) using the Volkov solution [8,9] to the Dirac equation. The electron effective mass in a strong PWF is constant, although deviating from that

\footnotetext{
*erez.raicher@mpi-hd.mpg.de
}

Published by the American Physical Society under the terms of the Creative Commons Attribution 4.0 International license. Further distribution of this work must maintain attribution to the author(s) and the published article's title, journal citation, and DOI. Open access publication funded by the Max Planck Society. in vacuum. The latter is characterized by the classical strong field parameter $\xi \equiv e a / m[7,10]$, where $a$ is the amplitude of the laser vector potential $A_{\mu}$, and $-e$ and $m$ are the electron charge and mass, respectively; relativistic units $\hbar=c=1$ are used. Contemporary optical lasers $[11,12]$ may reach $\xi \sim$ 100 (corresponding to an intensity of $\sim 10^{22} \mathrm{~W} / \mathrm{cm}^{2}$ ) and a significant increase is expected in next-generation laser facilities $[13,14]$. In addition to the field amplitude, the effective mass was shown to depend on the laser polarization [7] and pulse shape [15-20].

In the realm of strong-field QED, the perturbation treatment is developed based on solutions of the Dirac equation in the presence of an external field [21]. The fundamental quantity of this theory is the quantum strong-field parameter $\chi \equiv e \sqrt{-\left(F^{\mu v} P_{v}\right)^{2}} / m^{3}$ [7], where $P_{v}=(\mathcal{E}, \mathbf{P})$ is the kinetic four-momentum, a bold letter stands for a 3-vector, and $F_{\mu \nu}=$ $\partial_{\mu} A_{v}-\partial_{v} A_{\mu}$ is the field tensor. The lowest-order processes described by this theory are the Schwinger mechanism [22], where the strong field induces a pair creation from the vacuum, the nonlinear Compton (NLC), where an electron absorbs $s$-laser photons to emit an energetic photon $[7,10]$, and the nonlinear Breit-Wheeler (NLBW), where an electron positron pair is created following the absorption of a $\gamma$ photon and $s$-laser photons. The effective mass is manifested in the kinematics associated with these processes and thus may be measured. The first process is exponentially inhibited for fields below the Schwinger field $E_{s} \equiv m^{2} / e$ (corresponding to an intensity of $\sim 10^{29} \mathrm{~W} / \mathrm{cm}^{2}$ for optical lasers) and is beyond experimental reach in the near future [10]. The second and third scatterings are closely related through the crossing symmetry [2] and are assumed to play a key role in laser-matter interactions [23-30]. In the classical regime $(\chi \ll 1)$, only the NLC has an analog (the nonlinear Thomson scattering). In this domain, which is accessible to experimental investigations, the effective mass was recently measured [31,32]. In the quantum regime both the NLC and the NLBW were observed in the E-144 experiment [33,34], but the effective mass was 
not directly detected. Recently, however, several experiments aiming at strong-field QED were reported [35-38], bringing the perspective closer to measuring the effective mass in the quantum domain as well.

In strong laser fields, the potential experienced by the particle is periodic and hence one could expect that the effectivemass features would resemble the condensed-matter case, inducing a complex dispersion relationship for the dressed electron. Nevertheless, through all previous results, the effective mass is always constant and no deviation from the simple dispersion was reported. At first sight surprising, this fact stems from the symmetry of PWF, namely, from the specific space-time dependence of the field via $t-z$. Nevertheless, the modern ultrastrong laser technique frequently employs more complex electromagnetic configurations, e.g., a combination of several laser beams [39-41], which may induce more complicated dispersion relations.

The simplest example of a multibeam laser field configuration is the case of two counterpropagating laser pulses. The antinodes of the electric field in this case can be modeled by an oscillating electric field. Several approximations to the corresponding wave function were discussed in the context of various strong-field processes [42-54]. The effective mass and its consequences, however, were explicitly considered only for the limiting case of a vanishing particle momentum [55], and no deviation of the effective mass from the simple dispersion was revealed.

In this paper, the example of a rotating electric field (REF) is employed to show that for external fields deviating from the PWF, a momentum-dependent effective mass may arise. We derive the analytic expression for the effective mass of a particle in the presence of REF and show that it depends not only on the field parameters but also on the particle momentum. Namely, two particles propagating in different directions or velocities in the same field acquire a different mass. The effects of the dressed mass on the probabilities of NLC and NLBW processes, and on the spectra of the emitted photons and the created electron-positron pairs, are explored by analytical and numerical means. Thus, signatures of a nontrivial dispersion relation, analogous to condensedmatter systems, are explicitly manifested in fundamental QED scattering processes. Furthermore, two experimental scenarios are suggested to detect a measurable signature of this phenomenon.

This paper is organized as follows. In Sec. II, the effective mass in the presence of a REF is analyzed. Section III presents the various signatures of the effective mass appearing in NLC and NLBW processes and proposes an experimental setup where they can be measured. Section IV discusses the physical consequences of the obtained results and concludes the paper.

\section{THE EFFECTIVE MASS}

First, let us explicitly calculate the effective mass of a particle in the field under consideration. The effective mass is defined via the cycle-averaged momentum of the electron in this field: $m_{*} \equiv \sqrt{\bar{P}^{2}}$. The vector potential of REF is defined as $A^{\mu}=a_{1}^{\mu} \cos (\omega t)+a_{2}^{\mu} \sin (\omega t)$, where $a_{1}^{\mu}=a(0,1,0,0)$, $a_{2}^{\mu}=a(0,0,1,0)$ are the polarization vectors, and the $(x-y)$ plane is the polarization plane. The time-dependent momentum reads $\mathbf{P}=\mathbf{p}-e \mathbf{A}$, with the initial momentum $p_{\mu}=$ $(\varepsilon, \mathbf{p})$, and the time-dependent energy is derived from the free-electron dispersion relation $\mathcal{E}=\sqrt{m^{2}+\mathbf{P}^{2}}$. Without loss of generality we assume that the particle propagates in the $(x-z)$ plane, so that $\mathbf{p}=p(\sin \theta, 0, \cos \theta)$, where $p \equiv|\mathbf{p}|$ and $\theta$ is the angle between $\mathbf{p}$ and the $z$ axis, transverse to the polarization plane. The cycle-average momentum is simply $\mathbf{p}$ and the cycle-average energy is given by $\overline{\mathcal{E}}=$ $2 G E_{2}(\mu) / \pi$ [45], where $E_{2}$ is the elliptic integral of the second kind, $\mu \equiv 4 m \xi p|\sin \theta| / G^{2}$, and $G \equiv\left[m^{2}\left(1+\xi^{2}\right)+\right.$ $\left.p^{2}+2 m \xi p|\sin \theta|\right]^{1 / 2}$. Hence, the effective mass takes the form

$$
m_{*}=\sqrt{\left[\frac{2 G E_{2}(\mu)}{\pi}\right]^{2}-\mathbf{p}^{2}} .
$$

Generally speaking, it depends on three quantities: $\xi, p$, and $\theta$. In the following, we examine analytically its limits. For a particle initially propagating perpendicular to the field plane, i.e., $\theta=0$, as well as for $p=0$, one obtains $\mu=0$. Consequently, since $E_{2}(0)=\pi / 2$, the effective mass recovers its PWF value $m_{*}=m_{*}^{P} \equiv m \sqrt{1+\xi^{2}}$. It coincides with the result of Ref. [55], which was obtained for vanishing momentum, and agrees with Ref. [51], obtained for a head-on collision $(\theta=0)$. An explanation of this fact is suggested later on. In the case of $p \ll m \xi$, one obtains $\mu \ll 1$. Since the first-order Taylor expansion of $E_{2}$ with respect to $\mu$ vanishes, this limit corresponds, up to second order, to $m_{*} \approx m_{*}^{P}$. For the opposite case $(p \gg m \xi)$, one may expand $G$ and $E_{2}$ (see Appendixes A and B) appearing in the general expression, which leads to

$$
m_{*} \approx m_{*}^{P} \sqrt{1-\frac{\xi^{2}}{2\left(1+\xi^{2}\right)} \sin ^{2} \theta} .
$$

Accordingly, the minimal value of $m_{*}$, corresponding to $\xi \gg 1$, is $m_{*} \approx m_{*}^{P} / \sqrt{2}$. In this limit, however, the local crossed-field approximation sets in for the NLC and NLBW processes, when the probabilities and spectra depend solely on the parameter $\chi$, but not on $\xi$, and all signatures of the effective mass vanish. Thus, the preferable range for the study of the effective-mass influence is $\xi \sim 1$. Figure 1(a) shows the effective mass for $\xi=2$ (normalized to the PWF value
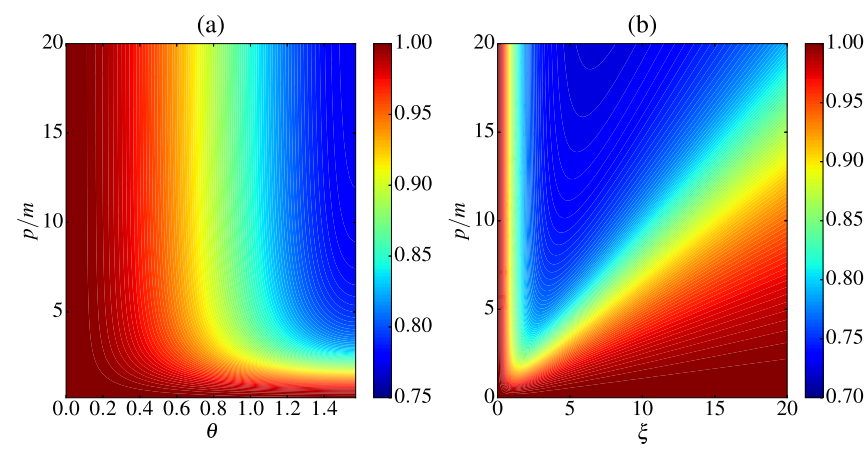

FIG. 1. The effective mass $m_{*}$, normalized to the PWF value $m_{*}^{P}$ : (a) as a function of $p / m$ and $\theta$ for $\xi=2$; (b) as a function of $p / m$ and $\xi$ for $\theta=\pi / 2$. 
$\left.m_{*}^{P}\right)$ as a function of $\theta$ and $p / m$. One may observe that for $\theta=0$ or $p \ll m \xi$ the normalized value of the effective mass tends to 1 , in agreement with the analytical result. The values for $p \gg m \xi$ coincide to a very good approximation with Eq. (2). Figure 1(b) presents the same quantity as a function of $p / m$ and $\xi$ for $\theta=\pi / 2$. The limits of $p$ either much higher or much lower than $m \xi$ hold here as in Fig. 1(a). Notice that the minimal value of the normalized effective mass is $1 / \sqrt{2} \approx 0.71$ and that a significant decrease appears for $p \gg m \xi, \xi \sim 1$, in accordance with Eq. (2).

\section{SIGNATURES IN STRONG-FIELD QED PROCESSES}

\section{A. Calculation of probabilities and a proposed setup}

Since the effective mass is embedded in the kinematics associated with the NLC and NLBW processes, its fingerprint may be found in the corresponding spectra. In a previous work [54] we have examined in detail the NLC probability for this field configuration. It was found that as long as $\varepsilon \gg m \xi$, the rate coincides to an excellent approximation with the one obtained with the semiclassical formula introduced by Baier and Katkov [56,57]. Due to the crossing symmetry between the matrix elements of the Compton and Breit-Wheeler processes, this conclusion holds for the NLBW process as well. For this reason, we calculate here the rate according to the semiclassical expression. In this case, the probability to emit a photon with a four-momentum $k^{\prime}=\left(\omega^{\prime}, \mathbf{k}^{\prime}\right)$ reads

$$
d \mathcal{P}=\frac{\alpha}{(2 \pi)^{2} \omega^{\prime}}\left[-\frac{\varepsilon^{\prime 2}+\varepsilon^{2}}{2 \varepsilon^{\prime 2}}\left|\mathcal{T}_{\mu}\right|^{2}+\frac{m^{2} \omega^{\prime 2}}{2 \varepsilon^{\prime 2} \varepsilon^{\prime 2}}|\mathcal{I}|^{2}\right] d^{3} \mathbf{k}^{\prime},
$$

where $\alpha \approx 1 / 137$ is the fine-structure constant and $\varepsilon^{\prime}=\varepsilon-$ $\omega^{\prime}$. The integrals $\mathcal{I}$ and $\mathcal{T}_{\mu}$ are defined as follows, $\mathcal{I} \equiv$ $\int_{-\infty}^{\infty} d t e^{i \psi}$ and $\mathcal{T}_{\mu} \equiv \int_{-\infty}^{\infty} d t v_{\mu} e^{i \psi}$, where the phase reads $\psi \equiv \frac{\varepsilon}{\varepsilon^{\prime}} k^{\prime} \cdot x(t)$, the velocity is $v_{\mu}=P_{\mu} / \mathcal{E}$, and $x(t)$ designates the classical trajectory. The probability associated with the NLBW takes an analogous form where $d^{3} \mathbf{k}^{\prime}$ is replaced by the momentum of the outgoing electron $d^{3} \mathbf{p}^{\prime}$ and $\varepsilon^{\prime}=\omega^{\prime}-\varepsilon$. The derivation of the final expressions for the two processes is given in Appendixes C and D. It follows from Eq. (3) that the probability is determined according to the trajectory of the electron in the presence of the field. It provides an explanation for the fact that for $\theta=0$ the effective mass coincides with that of the PWF, as seen from Eq. (2). In this case, the particle is simply moving in a circle in the $(x-y)$ plane while drifting along the $z$ axis, which is identical to the particle motion in a PWF.

A possible realization of REF in the laboratory may be achieved using counterpropagating circularly polarized laser beams, as illustrated schematically in Fig. 2. In the antinodes of the standing wave created by the beams, the magnetic components of the two beams cancel each other and the field can be approximated as REF. As a result, a particle would experience REF rather than a standing wave only if it propagates along the antinode plane (perpendicular to the beam axis), namely, with $\theta=\pi / 2$. On the other hand, we wish to detect the angle dependence of the effective mass. According to Fig. 1, this dependence is slow and monotonous. Thus, finding another configuration corresponding to $\theta=0$ may be sufficient. As explained above, the latter case is theoretically

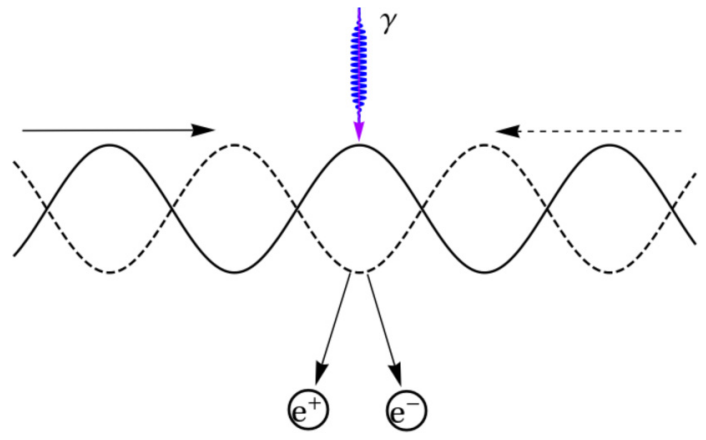

FIG. 2. The schematic setup. The counterpropagating beams create a standing wave and the $\gamma$-photon beam passes through the antinode and creates electron-positron pairs.

equivalent to a particle in the presence of a PWF. Hence, our reference configuration would be a $\gamma$ photon interacting with a circularly polarized PWF with the same $\xi$ value. Since for the PWF the effective mass depends solely on $\xi$, the angle between the $\gamma$ photon and the laser may be chosen according to convenience. In the following, we assumed that this angle would be $\theta=\pi / 2$. Namely, the reference configuration is identical to the one presented in Fig. 2, where the two beams are replaced by a single one with the same total energy.

\section{B. Nonlinear Breit-Wheeler}

For the NLBW process to take place, the center-of-mass energy $E_{s}=\sqrt{\left(s k+k^{\prime}\right)^{2}}=\sqrt{2 s\left(k \cdot k^{\prime}\right)}$ should exceed $2 m_{*}$, where $s$ is the number of absorbed field quanta and their wave vector reads $k=(\omega, 0,0,0)$. This threshold suggests a simple way to measure the effective mass. Since for the setup illustrated in Fig. 2 we have $k \cdot k^{\prime}=\omega \omega^{\prime}$, the threshold energy for the incoming $\gamma$ photon is

$$
\omega_{s}^{\prime}=\frac{2 m_{*}^{2}}{s \omega} .
$$

Accordingly, increasing $\omega^{\prime}$ for fixed laser parameters leads to a discrete change in the number of allowed channels in the vicinity of $\omega_{s}^{\prime}$, leading to an abrupt jump in the total probability. In order to detect this discontinuity, two requirements should be fulfilled. First, the laser normalized amplitude should lay in the perturbative regime (i.e., $\xi \lesssim 1$ ), so that high harmonics are inhibited and the main contribution originates from the $s$ th channel under consideration. Second, the threshold $\omega_{s}^{\prime}$ should be remote from the sequential one $\omega_{s+1}^{\prime}$, so that the influence of the sth channel would be distinguishable. Therefore, as Eq. (4) implies, low harmonics are preferable. The total probability of pair production in dependence of the incoming $\gamma$-photon energy is shown in Fig. 3(a). Since high $\omega^{\prime}$ of $\gamma$-photon energies are difficult to achieve, we propose to increase $\omega$ by using harmonics of the laser radiation, and consider the following laser parameters: $\xi=0.4, \omega=$ $4.65 \mathrm{eV}$, corresponding to the third harmonic of a Ti:S laser with an intensity of $6 \times 10^{18} \mathrm{~W} / \mathrm{cm}^{2}$. As mentioned above, observing effective-mass effects requires a periodic laser field, namely, a multicycle pulse. A 10-cycle pulse with the desired intensity focused on a spot with a diameter of 10 wavelengths corresponds to $4 \mathrm{~mJ}$, which is realizable with the present 

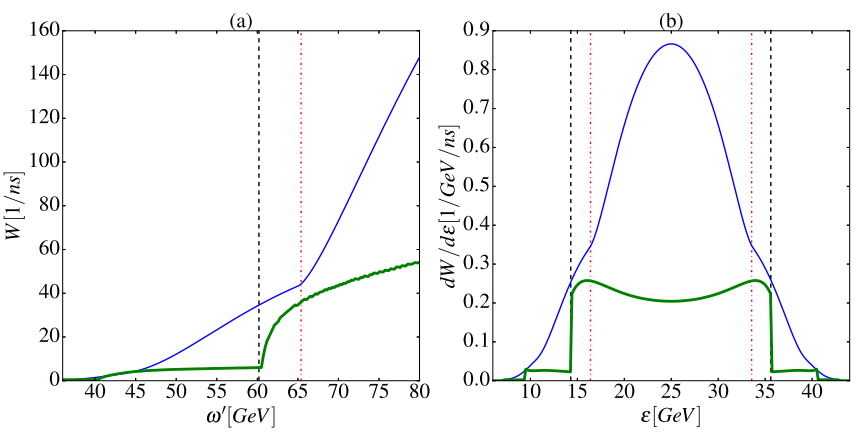

FIG. 3. (a) Total pair production probability vs incoming $\gamma$ photon energy: $\theta=0$ (thin blue line) and $\theta=\pi / 2$ (green line). The vertical dashed-dotted red (dashed black) line shows the threshold location $\omega_{2}^{\prime}$ for $\theta=0(\theta=\pi / 2)$. (b) Energy spectrum of the emitted pairs for the $\gamma$-photon energy of $\omega^{\prime}=50 \mathrm{GeV}: \theta=0$ (thin blue line) and $\theta=\pi / 2$ (green line). The vertical dashed-dotted red (dashed black) lines show the location of third harmonic edge for $\theta=0$ $(\theta=\pi / 2)$. The laser parameters are $\omega=4.65 \mathrm{eV}, \xi=0.4$.

laser technique [58]. The $\gamma$ energies lie in the same $\mathrm{GeV}$ range as those achieved in the E-144 experiment $[33,34]$. One may observe that the thresholds are $\omega_{2}^{\prime}=65.2 \mathrm{GeV}$ for $\theta=0$, and $60.5 \mathrm{GeV}$ for $\theta=\pi / 2$, which by using Eq. (4) correspond to $m_{*}(\theta=0)=m_{*}^{P}$ and $m_{*}(\theta=\pi / 2)=0.96 m_{*}^{P}$, in accordance with Eq. (2). Notice that for $\theta=0$ the quantum parameter is $\chi=\xi \omega \omega^{\prime} / m^{2}$ whereas for $\theta=\pi / 2$ it reads $\xi \omega \omega^{\prime} / m^{2} \sin (\omega t)$. Accordingly, the average value of $\chi$ is lower in the second case and so is the corresponding rate.

Another indication of the effective mass may be observed in the spectrum of the created pair, as follows. From the energy momentum conservation $s k_{\mu}+\bar{P}_{\mu}=\bar{P}_{\mu}^{\prime}+k^{\prime}$, a restriction on the outgoing particle energy arises (see Appendix D). For a given number of absorbed photons $s$, one may show that

$$
\left|\varepsilon-\frac{\omega^{\prime}}{2}\right|<\frac{\Delta_{s}}{2}, \quad \Delta_{s}=\omega^{\prime} \sqrt{1-\frac{s_{0}}{s}},
$$

where $s_{0}=2 m_{*}^{2} /\left(\omega \omega^{\prime}\right)$. As an example, the spectral probability associated with the created pair is depicted in Fig. 3(b). The $\gamma$-photon energy is $50 \mathrm{GeV}$ and the laser parameters as described above. The widths of the third harmonic are $\Delta_{3}=$ $0.34 \omega^{\prime}, 0.43 \omega^{\prime}$ for $\theta=0, \pi / 2$, respectively. Employing (5), one obtained the same effective-mass values written above.

\section{Nonlinear Compton}

In the following, a similar analysis is carried out regarding the NLC process (the PWF case was discussed in Ref. [23]). A straightforward kinematic calculation (see Appendix C) shows that for a given $s$, the emitted photon has a cutoff energy, known as an "edge,"

$$
\omega_{e}^{\prime}=\frac{s \omega \varepsilon}{\varepsilon(1-\bar{v})+s \omega}, \quad \bar{v}=\frac{p}{\sqrt{m_{*}^{2}+p^{2}}},
$$

where $\bar{v}$ is the absolute value of the cycle-averaged velocity. As a result, the effective mass affects the edge location. In principle, since the effective mass is momentum dependent (as shown in Fig. 1), it may differ for the incoming and outgoing particles. We study the process in the classical regime,
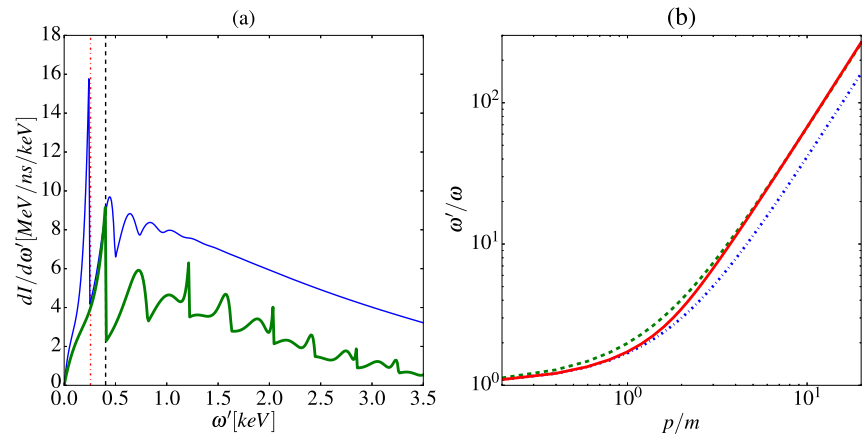

FIG. 4. (a) NLC emission spectrum: $\theta=0$ (thin blue line) and $\theta=\pi / 2$ (green line). Simulation parameters: $\omega=1.55 \mathrm{eV}, \xi=2$, $p / m=20$. The vertical dashed-dotted red (dashed black) line shows the edge location of the first harmonic $\theta=0(\theta=\pi / 2)$. (b) The edge energy for $\theta=\pi / 2$, normalized by $\omega$, as a function of $p / m$ (red line). As a reference, the prediction of Eq. (6) for the limiting cases $m_{*}(p=0)$ (dashed-dotted blue line) and $m_{*}(p \gg m \xi)$ (dashed green line).

$\chi \ll 1$, because the regime where both $\xi \sim 1$ and $\chi \sim 1$ are fulfilled would require very high-frequency colliding laser beams (with photon energies in the $\mathrm{MeV}$ range, which is beyond contemporary experimental reach). As a result, the recoil is negligible $\left(p \approx p^{\prime}\right)$ and therefore the effective masses of the incoming and outgoing electron are the same. The emission properties of a particle propagating in the electric field plane may be measured in a setup similar to the one in Fig. 2, where the $\gamma$ photons are replaced by high-energy electrons. Aiming the electron beam at the antinode may be more challenging as compared to the previous case as a result of the pondermotive force acting on the electrons. However, the intensity considered is moderate, allowing for a comparatively large spot, weak gradients, and therefore reduced pondermotive force. A notable fact is that the NLC process has no threshold, as opposed to the NLBW discussed above. Namely, all possible channels $s$ are allowed, regardless of the incoming electron energy. Consequently, the first harmonic of the Ti:S laser as well as a modest electron energy are sufficient. Furthermore, the NLC spectrum is less sensitive to an increase in the field amplitude $\xi$ as compared to the NLBW one. As a result, one may use higher values of $\xi$ without losing the edge structure. These two facts allow one to explore the edge structure and thus the effective mass for $p \sim m \xi$. In this regime, as opposed to the $p \gg m \xi$ case discussed above, the effective mass depends not only on $\theta$ but on $p$ as well (see Fig. 1). Figure 4(a) presents the NLC spectrum for $\theta=0$ and $\theta=\pi / 2$. The laser frequency and intensity are $\omega=1.6 \mathrm{eV}$, $I=1.7 \times 10^{19} \mathrm{~W} / \mathrm{cm}^{2}$, corresponding to $\xi=2$. The particle initial momentum is $p / m=20$. One may see that the harmonics edges become smeared with increasing $s$. As a result, it is convenient to take a closer look at the first harmonic only. The edge locations corresponding to $\theta=0$ and $\theta=\pi / 2$ are $\omega_{e}^{\prime}=$ $0.26 \mathrm{keV}$ and $\omega_{e}^{\prime}=0.4 \mathrm{keV}$, respectively. From Eq. (6) one may calculate the effective masses $m_{*}(\theta=0)=m_{*}^{P}, m_{*}(\theta=$ $\pi / 2)=0.77 m_{*}^{P}$, in agreement with the prediction of Eq. (2). As in the NLBW case, the average value of $\chi$ is smaller for $\theta=\pi / 2$, leading to a lower spectrum. By varying the incoming electron momentum one may observe the shift of the 
edge location. The shift of the edge from the calculated spectra is summarized in Fig. 4(b). From the latter the effective mass is deduced using Eq. (6), which is in accordance with the function $m_{*}(\pi / 2, p / m)$ presented in Fig. 1(a) and calculated from the classical trajectory. As a reference, the predictions of Eq. (6) for constant effective-mass values corresponding to the limiting cases $p=0$ and $p \gg m \xi$ are shown in Fig. 4(b) as well. As expected, the curve obtained from the edge location shift (solid red line) interpolates continuously between the two other ones.

\section{SUMMARY AND CONCLUSIONS}

In this paper, the effective mass in the presence of a REF was investigated in detail. It was shown to depend not only on the field amplitude but also on the particle momentum and propagation direction. The influence of this effect on the probabilities of the NLBW and NLC scatterings was explored by employing the Baier-Katkov semiclassical formalism. For the NLBW process, it was found that due to the angle dependence of the effective mass, the threshold for pair production varies with the angle of the $\gamma$ photon with respect to the field plane. Furthermore, the edge corresponding to a certain harmonic is determined according to the effective mass. Hence, its angle dependence may be inferred from the created pair spectrum. An analogous kinematic relation holds for the NLC process as well, allowing one to measure the effective mass by the location of the harmonics edges. In addition, since an energetic threshold does not exist, the incoming electron momentum may be chosen to be not much higher than the field amplitude, $p \gtrsim m \xi$. Accordingly, one may also examine experimentally the influence of the absolute value of the momentum (rather than its angle) on the effective mass.

The primary significance of the above results lies in the emergence of a nontrivial dispersion relation in the context of strong-field QED, namely, the demonstration of high-energy scattering processes of dressed particles with a dispersion relation resembling those observed in condensed-matter systems, rather than that of free particles. It implies that more complex electromagnetic configurations (e.g., Refs. [39-41]) may induce more complicated dispersion relations, which may be manipulated by the characteristics of the beams.

\section{ACKNOWLEDGMENTS}

E.R. is grateful to Q. Z. Lyu for helpful discussions. E.R. acknowledges support from the Alexander von Humboldt Foundation.

\section{APPENDIX A: THE CLASSICAL TRAJECTORY}

In the semiclassical formalism employed in this paper, the classical trajectory of the particle is the cornerstone of the rate calculation. Let us calculate explicitly the trajectory of an electron moving in a rotating electric field (REF), described by a vector potential $\mathbf{A}(t)=a(\cos \omega t, \sin \omega t, 0)$. The particle location is given by

$$
\mathbf{x}(t)=\int_{t_{0}}^{t} d t^{\prime} v\left(t^{\prime}\right)=\int_{t_{0}}^{t} d t^{\prime} \frac{\mathbf{P}\left(t^{\prime}\right)}{\mathcal{E}\left(t^{\prime}\right)} .
$$

Due to the canonical momentum conservation, the kinetic momentum is straightforwardly derived,

$$
\mathbf{P}(t)=\mathbf{p}+e \mathbf{A}(t)
$$

and using the dispersion relationship $\mathcal{E}(t)=\sqrt{\mathbf{P}^{2}(t)+m^{2}}$, one arrives at

$$
\mathcal{E}(t)=\sqrt{\varepsilon_{0}^{2}+(e a)^{2}+2 e a p|\sin \theta| \cos (\omega t-v)},
$$

where $\tan v=p_{y} / p_{x}$. It may be represented as

$$
\mathcal{E}=G \sqrt{1-\mu \sin ^{2}\left(\frac{\omega t-v}{2}\right)}
$$

where the following quantities are introduced,

$$
G \equiv \sqrt{m^{2}\left(1+\xi^{2}\right) p^{2}+2 m \xi p|\sin \theta|}
$$

and

$$
\mu \equiv \frac{4 m \xi p|\sin \theta|}{G^{2}}
$$

Substituting the explicit expression for the energy into Eq. (A1), one obtains the particle coordinate. Its $x$ component reads

$$
\begin{aligned}
x(t)= & \frac{1}{G} \int_{t_{0}}^{t} d t^{\prime}\left[\frac{p_{x}}{\sqrt{1-\mu \sin ^{2}\left(\frac{\omega t^{\prime}-v}{2}\right)}}\right. \\
& \left.+\frac{e a \cos \left(\omega t^{\prime}\right)}{\sqrt{1-\mu \sin ^{2}\left(\frac{\omega t^{\prime}-v}{2}\right)}}\right] .
\end{aligned}
$$

A variable change $\phi \equiv(\omega t-v) / 2$ yields

$$
x(t)=\frac{2}{\omega G} \int_{\phi_{0}}^{\phi} d \phi^{\prime}\left[\frac{p_{x}}{\sqrt{1-\mu \sin ^{2} \phi^{\prime}}}+\frac{e a \cos \left(2 \phi^{\prime}+v\right)}{\sqrt{1-\mu \sin ^{2} \phi^{\prime}}}\right] .
$$

The latter takes the form

$$
x(t)=\frac{2}{\omega G}\left[p_{x} \mathcal{J}_{1}+m \xi\left(\cos \nu \mathcal{J}_{2}-\sin \nu \mathcal{J}_{3}\right)\right]
$$

where the following integrals are defined,

$$
\begin{aligned}
& \mathcal{J}_{1}(x \mid \mu) \equiv \int_{0}^{x} d x^{\prime} \frac{1}{\sqrt{1-\mu \sin ^{2} x^{\prime}}}, \\
& \mathcal{J}_{2}(x \mid \mu) \equiv \int_{0}^{x} d x^{\prime} \frac{\cos \left(2 x^{\prime}\right)}{\sqrt{1-\mu \sin ^{2} x^{\prime}}}, \\
& \mathcal{J}_{3}(x \mid \mu) \equiv \int_{0}^{x} d x^{\prime} \frac{\sin \left(2 x^{\prime}\right)}{\sqrt{1-\mu \sin ^{2} x^{\prime}}},
\end{aligned}
$$


and $x=\omega t / 2$. These integrals admit an analytical solution,

$$
\begin{gathered}
\mathcal{J}_{1}(x \mid \mu)=E_{1}(x \mid \mu), \\
\mathcal{J}_{2}(x \mid \mu)=\frac{(\mu-2) E_{1}(x \mid \mu)+2 E_{2}(x \mid \mu)}{\mu}, \\
\mathcal{J}_{3}(x \mid \mu)=\frac{2}{\mu}\left[1-\sqrt{1-\mu \sin ^{2} x}\right],
\end{gathered}
$$

where $E_{1}(x \mid \mu), E_{2}(x \mid \mu)$ are the incomplete elliptic integrals of the first and second kind, respectively,

$$
\begin{aligned}
& E_{1}(x \mid \mu) \equiv \int_{0}^{x} d x^{\prime} \frac{1}{\sqrt{1-\mu \sin ^{2} x^{\prime}}}, \\
& E_{2}(x \mid \mu) \equiv \int_{0}^{x} d x^{\prime} \sqrt{1-\mu \sin ^{2} x^{\prime}} .
\end{aligned}
$$

Analogously, for the $y$ component of the coordinate, one obtains

$$
y(t)=\frac{2}{\omega G} \int_{\phi_{0}}^{\phi} d \phi^{\prime}\left[\frac{p_{y}}{\sqrt{1-\mu \sin ^{2} \phi^{\prime}}}+\frac{e a \sin \left(2 \phi^{\prime}+v\right)}{\sqrt{1-\mu \sin ^{2} \phi^{\prime}}}\right],
$$

which reads

$$
y(t)=\frac{2}{\omega G}\left[p_{y} \mathcal{J}_{1}+m \xi\left(\sin v \mathcal{J}_{2}+\cos v \mathcal{J}_{3}\right)\right] .
$$

The vector potential has no $z$ component, so for $z(t)$ we simply have

$$
z(t)=\frac{2}{\omega G} p_{z} \mathcal{J}_{1}
$$

In addition to the trajectory, the average velocity is required as well for the purpose of rate calculation. Applying the definition $\bar{v}_{x} \equiv[x(T)-x(0)] / T$, where $T=2 \pi / \omega$, we obtain

$$
\bar{v}_{x}=\left.\frac{1}{\pi G}\left[p_{x} \mathcal{J}_{1}+m \xi\left(\cos v \mathcal{J}_{2}-\sin \nu \mathcal{J}_{3}\right)\right]\right|_{0} ^{\pi} .
$$

One may easily find

$$
\begin{gathered}
\left.\mathcal{J}_{1}(x \mid \mu)\right|_{0} ^{\pi}=2 E_{1}(\mu), \\
\left.\mathcal{J}_{2}(x \mid \mu)\right|_{0} ^{\pi}=\frac{2(\mu-2) E_{1}(\mu)+4 E_{2}(\mu)}{\mu}, \\
\left.\mathcal{J}_{3}(x \mid \mu)\right|_{0} ^{\pi}=0,
\end{gathered}
$$

where the complete elliptic integrals are given by $E_{1}(\mu)=$ $E_{1}\left(\frac{\pi}{2} \mid \mu\right), E_{2}(\mu)=E_{2}\left(\frac{\pi}{2} \mid \mu\right)$. Accordingly, the $x$ component of the average velocity takes the form

$\bar{v}_{x}=\frac{2}{\pi G}\left[p_{x} E_{1}(\mu)+m \xi \cos \nu\left(\frac{2 E_{2}(\mu)+(\mu-2) E_{1}(\mu)}{\mu}\right)\right]$.

(A25)

Similarly, the other components are given by

$\bar{v}_{y}=\frac{2}{\pi G}\left[p_{y} E_{1}(\mu)+m \xi \sin v\left(\frac{2 E_{2}(\mu)+(\mu-2) E_{1}(\mu)}{\mu}\right)\right]$

and

$$
\bar{v}_{z}=\frac{2}{\pi G} p_{z} E_{1}(\mu)
$$

\section{APPENDIX B: THE HIGH MOMENTUM LIMIT OF THE EFFECTIVE MASS}

As explained in the main text, the effective mass is defined as $\sqrt{\bar{P}^{2}}$. Since $\overline{\mathbf{P}}=\mathbf{p}$ we have

$$
m_{*}=\sqrt{\overline{\mathcal{E}}^{2}-\mathbf{p}^{2}} .
$$

The cycle-averaged energy is given by

$$
\overline{\mathcal{E}}=\frac{2}{\pi} G E_{2}(\mu)
$$

In the following, we would like to Taylor expand the effective mass for $p \gg m \xi$. As we show below, the first order vanishes and, therefore, we evaluate it up to second order. We introduce the following quantities,

$$
\delta \equiv \frac{4 m \xi p|\sin \theta|}{R^{2}}, \quad R \equiv \sqrt{m^{2} \xi^{2}+m^{2}+p^{2}} .
$$

$G, \mu$ defined above read in terms of these variables:

$$
\begin{gathered}
G=R \sqrt{1+\frac{\delta}{2}}, \\
\mu=\frac{\delta}{1+\delta / 2} .
\end{gathered}
$$

Substituting Eqs. (B5) and (B4) into Eq. (B2), one finds

$$
\overline{\mathcal{E}}=\frac{2 R}{\pi} \sqrt{1+\frac{\delta}{2}} E_{2}\left(\frac{\delta}{1+\delta / 2}\right) .
$$

One may notice that up to third order,

$$
\delta \approx 4|\sin \theta|\left(\frac{m \xi}{p}\right)+O\left(\left[\frac{m \xi}{p}\right]^{3}\right) .
$$

As a result, up to second order, we may expand with respect to $\delta$ instead of $m \xi / p$. Employing the following Taylor expansions,

$$
\begin{gathered}
E_{2}(x) \approx \frac{\pi}{2}\left(1-\frac{x}{4}-\frac{3 x^{2}}{64}\right), \\
\sqrt{1+x} \approx 1+\frac{x}{2}-\frac{x^{2}}{8},
\end{gathered}
$$

and substituting Eq. (B6) into Eq. (B1), one obtains

$$
m_{*}^{2}=\left(m_{*}^{P}\right)^{2}-\frac{\delta^{2} R^{2}}{32}
$$

where $m_{*}^{P}=m \sqrt{1+\xi^{2}}$. Since

$$
\delta^{2} R^{2}=\frac{16 m^{2} p^{2} \xi^{2} \sin ^{2} \theta}{R^{2}} \approx 16 m^{2} \xi^{2} \sin ^{2} \theta,
$$

one may see that Eq. (B10) becomes

$$
\frac{m_{*}}{m_{*}^{P}}=\sqrt{1-\frac{\xi^{2}}{2\left(1+\xi^{2}\right)} \sin ^{2} \theta} .
$$

\section{APPENDIX C: NONLINEAR COMPTON SCATTERING}

\section{Probability}

As demonstrated in Ref. [54], under the condition $\varepsilon \gg m \xi$, the quantum and semiclassical $[56,57]$ approaches coincide. 
Since the latter allows for a simpler calculation, it will be used in this paper. According to this approximation, the probability of a Dirac particle to emit a photon with a four-momentum $k^{\prime}$ is given by

$$
\begin{aligned}
d \mathcal{P}= & \frac{\alpha}{(2 \pi)^{2}} d^{3} \mathbf{k}^{\prime} \int_{-\infty}^{\infty} d t_{1} \int_{-\infty}^{\infty} d t_{2} N_{21} \\
& \times \exp \left[i \frac{\varepsilon}{\varepsilon-\omega^{\prime}} k^{\prime} \cdot\left[x\left(t_{1}\right)-x\left(t_{2}\right)\right]\right],
\end{aligned}
$$

where $x_{\mu}=[t, \mathbf{x}(t)]$ and $\mathbf{x}(t)$ is the particle classical trajectory found above. The prefactor is given by

$$
N_{21} \equiv \frac{1}{2 \varepsilon^{\prime 2}}\left[\left(\varepsilon^{\prime 2}+\varepsilon^{2}\right)\left[v\left(t_{1}\right) \cdot v\left(t_{2}\right)-1\right]+\frac{\omega^{\prime 2} m^{2}}{\varepsilon^{2}}\right] .
$$

It should be mentioned that this expression already contains averaging over the incoming electron spin and summing over the outgoing electron (photon) spin (polarization), respectively. Since $v\left(t_{1}\right) v\left(t_{2}\right)-1=-v_{\mu}\left(t_{1}\right) v^{\mu}\left(t_{2}\right)$, Eq. (C1) can be cast in the following form,

$$
d \mathcal{P}=\frac{\alpha}{(2 \pi)^{2}}|\mathcal{K}|^{2} d^{3} k^{\prime},
$$

where

$$
|\mathcal{K}|^{2} \equiv-\left(\frac{\varepsilon^{\prime 2}+\varepsilon^{2}}{2 \varepsilon^{\prime 2}}\right)\left|\mathcal{T}_{\mu}\right|^{2}+\frac{m^{2} \omega^{\prime 2}}{2 \varepsilon^{\prime 2} \varepsilon^{2}}|\mathcal{I}|^{2},
$$

with

$$
\mathcal{I} \equiv \int_{-\infty}^{\infty} d t e^{i \psi}, \quad \mathcal{T}_{\mu} \equiv \int_{-\infty}^{\infty} d t v_{\mu}(t) e^{i \psi}
$$

and

$$
\psi=\frac{\varepsilon}{\varepsilon^{\prime}} k^{\prime} \cdot x(t)=\frac{\varepsilon \omega^{\prime} t}{\varepsilon^{\prime}}\left(1-\mathbf{x} \cdot \mathbf{n}^{\prime}\right)
$$

where $k_{\mu}^{\prime}=\omega^{\prime}\left(1, \mathbf{n}^{\prime}\right)$. Since we are dealing with a periodic motion, the phase may be decomposed into a periodic and nonperiodic parts $\psi=\psi_{p}+\psi_{n p} \omega t$ with

$$
\psi_{p}=\frac{\varepsilon}{\varepsilon^{\prime}} \mathbf{n}^{\prime} \cdot \mathbf{x}_{p}, \quad \psi_{n p}=\frac{\varepsilon \omega^{\prime}}{\varepsilon^{\prime}}\left(1-\bar{v} \cdot \mathbf{n}^{\prime}\right),
$$

where the periodic part of the trajectory is given by

$$
\mathbf{x}_{p}(t)=\mathbf{x}(t)-\bar{v} t .
$$

Since we assume that the incoming electron propagates along the $x$ axis, $p_{\mu}=\left(\varepsilon, p_{x}, 0,0\right)$, the emitted photon parametrization is defined accordingly,

$$
\mathbf{n}^{\prime}=\left(\cos \theta_{e}, \sin \theta_{e} \sin \varphi_{e}, \sin \theta_{e} \cos \varphi_{e}\right),
$$

where $\theta_{e}, \varphi_{e}$ are the polar and azimuthal angles with respect to the $x$ axis, respectively. Replacing the periodic part of the integrands by their Fourier series, the integrals are solved,

$$
\mathcal{T}^{\mu}=2 \pi \sum_{s} \mathcal{T}_{s}^{\mu} \delta\left(\Omega_{s}\right), \quad \mathcal{I}=2 \pi \sum_{s} \mathcal{I}_{s} \delta\left(\Omega_{s}\right),
$$

where the argument of the delta function reads

$$
\Omega_{s} \equiv \psi_{n p}-s \omega,
$$

and the Fourier coefficients are

$$
\begin{gathered}
\mathcal{T}_{s}^{\mu}=\frac{1}{T} \int_{0}^{T} d t v_{\mu}(t) e^{i\left(s \omega t-\psi_{p}\right)}, \\
\mathcal{I}_{s}=\frac{1}{T} \int_{0}^{T} d t e^{i\left(s \omega t-\psi_{p}\right)} .
\end{gathered}
$$

With the aid of the condition $\Omega_{s}=0$, forced by the delta functions, the angle $\theta_{e}$ is found,

$$
\cos \theta_{e}=\frac{1}{\bar{v}}\left(1-\frac{s \omega \varepsilon^{\prime}}{\omega^{\prime} \varepsilon}\right) .
$$

Using Eq. (C9), the periodic part of the phase takes the form

$$
\psi_{p}=\frac{\varepsilon \omega^{\prime}}{\varepsilon-\omega^{\prime}}\left[\cos \theta_{e} x_{p}(t)+\sin \theta_{e} \sin \varphi_{e} y_{p}(t)\right] .
$$

Substituting Eq. (C10) into Eq. (C4), and using the identity $\delta^{2}\left(\Omega_{s}\right)=\frac{\tau}{2 \pi} \delta\left(\Omega_{s}\right)$, with the interaction time $\tau$, one obtains

$$
|\mathcal{K}|^{2}=2 \pi \sum_{s} \mathcal{K}_{s}^{2} \delta\left(\Omega_{s}\right) \tau,
$$

where

$$
\left|\mathcal{K}_{s}\right|^{2}=-\left(\frac{\varepsilon^{\prime 2}+\varepsilon^{2}}{2 \varepsilon^{\prime 2}}\right)\left|\mathcal{T}_{s}^{\mu}\right|^{2}+\frac{m^{2} \omega^{\prime 2}}{2 \varepsilon^{\prime 2} \varepsilon^{2}}\left|\mathcal{I}_{s}\right|^{2} .
$$

Using $d^{3} \mathbf{k}^{\prime}=\omega^{\prime 2} d\left(\cos \theta_{e}\right) d \varphi_{e}$ and integrating Eq. (C3) over $\cos \theta_{e}$ yields

$$
\frac{d I}{d \omega^{\prime} d \varphi_{e}}=\frac{1}{(2 \pi)} \omega^{\prime 2} \sum_{s}\left|\mathcal{K}_{s}\right|^{2}\left|\frac{d \Omega_{s}}{d\left(\cos \theta_{e}\right)}\right|^{-1},
$$

where the relation $d I=\omega^{\prime} d \mathcal{P} / \tau$ between the probability and the radiation intensity was employed. Since $\bar{v} \cdot \mathbf{n}^{\prime}=\bar{v} \cos \theta_{e}$, from Eq. (C11) it follows that

$$
\left|\frac{d \Omega_{s}}{d\left(\cos \theta_{e}\right)}\right|=\frac{\varepsilon \omega^{\prime} \bar{v}}{\varepsilon^{\prime}} .
$$

Hence, the final expression takes the form

$$
\frac{d I}{d \omega^{\prime} d \varphi_{e}}=\frac{\alpha \omega^{\prime}}{4 \pi \varepsilon^{3} \varepsilon^{\prime}} \sum_{s}\left[-\varepsilon^{2}\left(\varepsilon^{2}+\varepsilon^{\prime 2}\right)\left|\mathcal{T}_{s}^{\mu}\right|^{2}+m^{2} \omega^{\prime 2}\left|\mathcal{I}_{s}\right|^{2}\right]
$$

\section{Kinematics}

The highest possible value of $\omega^{\prime}$ associated with a given harmonics $s$ may be derived from kinematic considerations. Using $\varepsilon^{\prime}=\varepsilon-\omega^{\prime}$, the emitted photon energy stems from the kinematic relation Eq. (C14),

$$
\omega^{\prime}=\frac{s \omega \varepsilon}{\varepsilon\left(1-\bar{v} \cos \theta_{e}\right)+s \omega} .
$$

The maximal value of $\omega^{\prime}$ corresponds to $\cos \theta_{e}=1$. This result may be derived by an alternative kinematic approach. The energy momentum conservation of this process reads

$$
\bar{P}_{\mu}+s k_{\mu}=\bar{P}_{\mu}^{\prime}+k_{\mu}^{\prime},
$$


where $k_{\mu}=(\omega, 0,0,0)$. Therefore, the spatial momentum conservation yields

$$
p_{\|}^{\prime}+k_{\|}^{\prime}=p, \quad p_{\perp}^{\prime}=-k_{\perp}^{\prime},
$$

where $\|$ and $\perp$ designate the parallel and transverse components of the momenta with respect to the incoming particle direction, respectively. Then the total outgoing momentum reads

$$
p^{\prime}=\sqrt{m^{2}+p_{\|}^{\prime 2}+p_{\perp}^{\prime 2}}=\sqrt{\omega^{\prime 2}+p^{2}-2 p \omega^{\prime} \cos \theta_{e}},
$$

where $k_{\|}^{\prime}=\omega^{\prime} \cos \theta_{e}$ and $k_{\perp}^{\prime}=\omega^{\prime} \sin \theta_{e}$. Substituting $p^{\prime}$ into the energy conservation equation

$$
\overline{\mathcal{E}}+s \omega=\omega^{\prime}+\sqrt{m_{*}^{2}+p^{\prime 2}},
$$

one obtains the energy of the emitted photon,

$$
\omega^{\prime}=\frac{2 s \omega \overline{\mathcal{E}}+s^{2} \omega^{2}}{2\left(\overline{\mathcal{E}}-p \cos \theta_{e}+s \omega\right)} .
$$

Recalling that $\overline{\mathbf{P}}=\mathbf{p}$, the absolute value of the average velocity is given by $\bar{v}=p / \overline{\mathcal{E}}$. As a result, we have

$$
\omega^{\prime}=\frac{s \omega \overline{\mathcal{E}}}{\overline{\mathcal{E}}\left(1-\bar{v} \cos \theta_{e}\right)+s \omega},
$$

where $s \omega \ll \overline{\mathcal{E}}$ was assumed. Approximating $\overline{\mathcal{E}} \approx \varepsilon$, one returns to Eq. (C21) given above.

\section{APPENDIX D: NONLINEAR BREIT-WHEELER PROCESS}

\section{Probability}

Owing to the crossing symmetry relating the NLC and NLBW processes [2,57], the semiclassical probability associated with the latter takes the form

$$
d \mathcal{P}=\frac{\alpha}{(2 \pi)^{2} \omega^{\prime}}|\mathcal{K}|^{2} d^{3} \mathbf{p} .
$$

The difference with respect to the photon emission expression of Eq. (C3) is the outgoing particle phase space, namely, $d^{3} \mathbf{k}^{\prime} \rightarrow d^{3} \mathbf{p}$. Therefore, the final result Eq. (C20) should be only multiplied by a factor $\varepsilon^{2} / \omega^{\prime 2}$. Moreover, in this case we are interested in the emission rate rather than intensity, leading to an additional $1 / \omega^{\prime}$ factor. Finally, one obtains

$\frac{d W}{d \varepsilon d \varphi_{e}}=\frac{\alpha}{4 \pi \varepsilon \varepsilon^{\prime} \omega^{\prime 2}} \sum_{s}\left[-\varepsilon^{2}\left(\varepsilon^{2}+\varepsilon^{\prime 2}\right)\left|\mathcal{T}_{s}^{\mu}\right|^{2}+m^{2} \omega^{\prime 2}\left|\mathcal{I}_{s}\right|^{2}\right]$,

where $\mathcal{T}_{s}^{\mu}, \mathcal{I}_{s}$ are given by Eqs. (C12) and (C13). Another modification with respect to NLC scattering lies in the periodic part of the phase. Since the incoming photon propagates along the $x$ axis, one may write $\mathbf{n}^{\prime}=(1,0,0)$. Therefore, the outgoing electron four-momentum is parametrized as

$$
p_{\mu}=\left(\varepsilon, p \cos \theta_{e}, p \sin \theta_{e} \sin \varphi_{e}, p \sin \theta_{e} \cos \varphi_{e}\right) .
$$

Accordingly, $\psi_{p}$ may be written as

$$
\psi_{p}=\frac{\varepsilon \omega^{\prime}}{\omega^{\prime}-\varepsilon} \cos \theta_{e} x_{p}(t),
$$

where the trajectory is given by Eq. (A9), and the relation $\varepsilon^{\prime}=$ $\omega^{\prime}-\varepsilon$ is used.

\section{Kinematics}

As in the NLC case, the effective mass may be inferred from the maximal value of the outgoing particle energy for a given harmonic $s$. The kinematic relation Eq. (C14), together with $\varepsilon^{\prime}=\omega^{\prime}-\varepsilon$, yields

$$
\cos \theta_{e}=\frac{1}{\bar{v}}\left[1-\frac{s \omega\left(\omega^{\prime}-\varepsilon\right)}{\omega^{\prime} \varepsilon}\right] .
$$

Since $\cos \theta_{e} \leqslant 1$ and employing

$$
1-\bar{v}=\frac{\varepsilon-\sqrt{\varepsilon^{2}-m_{*}^{2}}}{\varepsilon} \approx \frac{m_{*}^{2}}{2 \varepsilon^{2}},
$$

one obtains

$$
\frac{s \omega\left(\omega^{\prime}-\varepsilon\right)}{\omega^{\prime} \varepsilon} \geqslant \frac{m_{*}^{2}}{2 \varepsilon^{2}} .
$$

Solving the quadratic inequality for $\varepsilon$, one arrives at

$$
\left|\varepsilon-\frac{\omega^{\prime}}{2}\right| \leqslant \sqrt{1-\frac{s_{0}}{s}}
$$

where $s_{0}=2 m_{*}^{2} /\left(\omega \omega^{\prime}\right)$.
[1] P. W. Higgs, Phys. Rev. Lett. 13, 508 (1964).

[2] M. E. Peskin and D. V. Schroeder, An Introduction to Quantum Field Theory (Perseus Books, Reading, 1995).

[3] E. M. Lifshitz and L. P. Pitaevskii, Statistical Physics, Course in Theoretical Physics Vol. 9 (Pergamon, Oxford, UK, 1980).

[4] A. I. Nikishov and V. I. Ritus, Sov. Phys. JETP 19, 529 (1964).

[5] T. W. B. Kibble, Phys. Rev. 150, 1060 (1966).

[6] H. R. Reiss, Phys. Rev. A 89, 022116 (2014).

[7] V. I. Ritus, J. Sov. Laser Res. 6, 497 (1985).

[8] D. M. Wolkow, Z. Phys. 94, 250 (1935).

[9] V. B. Berestetskii, E. M. Lifshitz, and L. P. Pitaevskii, Quantum Electrodynamics, Course in Theoretical Physics Vol. 4 (Pergamon, Oxford, UK, 1982).

[10] A. Di Piazza, C. Müller, K. Z. Hatsagortsyan, and C. H. Keitel, Rev. Mod. Phys. 84, 1177 (2012).
[11] J. W. Yoon, C. Jeon, J. Shin, S. K. Lee, H. W. Lee, I. W. Choi, H. T. Kim, J. H. Sung, and C. H. Nam, Opt. Express 27, 20412 (2019).

[12] The Gemini laser, https://www.clf.stfc.ac.uk/Pages/Lasersystem-Gemini.aspx

[13] The Extreme Light Infrustructure (ELI), http://www.extremelight-infrastructure.eu

[14] The XCELS project, http://www.xcels.iapras.ru

[15] C. Harvey, T. Heinzl, A. Ilderton, and M. Marklund, Phys. Rev. Lett. 109, 100402 (2012).

[16] M. Boca and V. Florescu, Phys. Rev. A 80, 053403 (2009).

[17] F. Mackenroth and A. Di Piazza, Phys. Rev. A 83, 032106 (2011).

[18] D. Seipt and B. Kämpfer, Phys. Rev. A 83, 022101 (2011). 
[19] T. Heinzl, A. Ilderton, and M. Marklund, Phys. Lett. B 692, 250 (2010).

[20] T. Nousch, D. Seipt, B. Kämpfer, and A. I. Titov, Phys. Lett. B 715, 246 (2012).

[21] W. H. Furry, Phys. Rev. 81, 115 (1951).

[22] J. Schwinger, Phys. Rev. 82, 664 (1951).

[23] C. Harvey, T. Heinzl, and A. Ilderton, Phys. Rev. A. 79, 063407 (2009).

[24] A. R. Bell and J. G. Kirk, Phys. Rev. Lett. 101, 200403 (2008).

[25] I. V. Sokolov, N. M. Naumova, J. A. Nees, and G. A. Mourou, Phys. Rev. Lett. 105, 195005 (2010).

[26] A. M. Fedotov, N. B. Narozhny, G. Mourou, and G. Korn, Phys. Rev. Lett. 105, 080402 (2010).

[27] E. N. Nerush, I. Yu. Kostyukov, A. M. Fedotov, N. B. Narozhny, N. V. Elkina, and H. Ruhl, Phys. Rev. Lett. 106, 035001 (2011).

[28] T. Nakamura, J. K. Koga, T. Z. Esirkepov, M. Kando, G. Korn, and S. V. Bulanov, Phys. Rev. Lett. 108, 195001 (2012).

[29] A. Gonoskov, I. Gonoskov, C. Harvey, A. Ilderton, A. Kim, M. Marklund, G. Mourou, and A. Sergeev, Phys. Rev. Lett. 111, 060404 (2013).

[30] A. Gonoskov, A. Bashinov, S. Bastrakov, E. Efimenko, A. Ilderton, A. Kim, M. Marklund, I. Meyerov, A. Muraviev, and A. Sergeev, Phys. Rev. X 7, 041003 (2017).

[31] K. Khrennikov, J. Wenz, A. Buck, J. Xu, M. Heigoldt, L. Veisz, and S. Karsch, Phys. Rev. Lett. 114, 195003 (2015).

[32] Y. Sakai, I. Pogorelsky, O. Williams, F. O'Shea, S. Barber, I. Gadjev, J. Duris, P. Musumeci, M. Fedurin, A. Korostyshevsky et al., Phys. Rev. ST Accel. Beams 18, 060702 (2015).

[33] D. L. Burke, R. C. Field, G. Horton-Smith, J. E. Spencer, D. Walz, S. C. Berridge, W. M. Bugg, K. Shmakov, A. W. Weidemann, C. Bula et al., Phys. Rev. Lett. 79, 1626 (1997).

[34] C. Bamber, S. J. Boege, T. Koffas, T. Kotseroglou, A. C. Melissinos, D. D. Meyerhofer, D. A. Reis, W. Ragg, C. Bula, K. T. McDonald et al., Phys. Rev. D 60, 092004 (1999).

[35] G. Sarri et al., Nat. Commun. 6, 6747 (2015).

[36] K. Poder, M. Tamburini, G. Sarri, A. Di Piazza, S. Kuschel, C. D. Baird, K. Behm, S. Bohlen, J. M. Cole, D. J. Corvan, M. Duff, E. Gerstmayr, C. H. Keitel, K. Krushelnick, S. P. D. Mangles, P. McKenna, C. D. Murphy, Z. Najmudin, C. P. Ridgers, G. M. Samarin et al., Phys. Rev. X 8, 031004 (2018).

[37] J. M. Cole, K. T. Behm, E. Gerstmayr, T. G. Blackburn, J. C. Wood, C. D. Baird, M. J. Duff, C. Harvey, A. Ilderton,
A. S. Joglekar, K. Krushelnick, S. Kuschel, M. Marklund, P. McKenna, C. D. Murphy, K. Poder, C. P. Ridgers, G. M. Samarin, G. Sarri, D. R. Symes et al., Phys. Rev. X 8, 011020 (2018).

[38] T. N. Wistisen, A. Di Piazza, H. V. Knudsen, and U. I. Uggerhøj, Nat. Commun. 9, 795 (2018).

[39] S. S. Bulanov, V. D. Mur, N. B. Narozhny, J. Nees, and V. S. Popov, Phys. Rev. Lett. 104, 220404 (2010).

[40] I. Gonoskov, A. Aiello, S. Heugel, and G. Leuchs, Phys. Rev. A 86, 053836 (2012).

[41] A. Gonoskov, A. Bashinov, I. Gonoskov, C. Harvey, A. Ilderton, A. Kim, M. Marklund, G. Mourou, and A. Sergeev, Phys. Rev. Lett. 113, 014801 (2014).

[42] E. Brezin and C. Izykson, Phys. Rev. D 2, 1191 (1970).

[43] C. Cronstrom and M. Noga, Phys. Lett. A 60, 137 (1977).

[44] W. Becker, Physica A 87, 601 (1977).

[45] G. R. Mocken, M. Ruf, C. Müller, and C. H. Keitel, Phys. Rev. A 81, 022122 (2010).

[46] A. Blinne and H. Gies, Phys. Rev. D 89, 085001 (2014).

[47] L. F. Granz, O. Mathiak, S. Villalba-Chávez, and C. Müller, Phys. Lett. B 793, 85 (2019).

[48] S. Varro, Laser Phys. Lett. 10, 095301 (2013).

[49] E. Raicher and S. Eliezer, Phys. Rev. A 88, 022113 (2013).

[50] E. Raicher, S. Eliezer, and A. Zigler, Phys. Lett. B 750, 76 (2015).

[51] T. Heinzl, A. Ilderton, and B. King, Phys. Rev. D 94, 065039 (2016).

[52] E. Raicher, S. Eliezer, and A. Zigler, Phys. Rev. A. 94, 062105 (2016).

[53] F. Mackenroth, N. Kumar, N. Neitz, and C. H. Keitel, Phys. Rev. E 99, 033205 (2019).

[54] E. Raicher, S. Eliezer, C. H. Keitel, and K. Z. Hatsagortsyan, Phys. Rev. A 99, 052513 (2019).

[55] C. Kohlfürst, H. Gies, and R. Alkofer, Phys. Rev. Lett. 112, 050402 (2014).

[56] V. N. Baier and V. M. Katkov, Sov. Phys. JETP 26, 854 (1968).

[57] V. N. Baier, V. M. Katkov, and V. M. Strakhovenko, Electromagnetic Processes at High Energies in Oriented Single Crystals (World Scientific, Singapore, 1998).

[58] Y. Wang, S. Wang, A. Rockwood, B. M. Luther, R. Hollinger, A. Curtis, C. Calvi, C. S. Menoni, and J. J. Rocca, Opt. Lett. 42, 3828 (2017). 